\title{
A Prototype Geospatial Knowledge Graph for National Topographic Mapping
}

\author{
Dalia E. Varanka ${ }^{\mathrm{a}, *}$ \\ ${ }^{a}$ U.S. Geological Survey, Dalia E. Varanka-dvaranka@usgs.gov \\ * Corresponding author
}

Keywords: geospatial knowledge graph, national topographic data, semantic technology

\begin{abstract}
:
Knowledge graphs $(\mathrm{KG})$ are a virtual layer connecting disparate databases into an interoperable framework. Though the application of KGs for enterprises are increasing, geospatial KG design is not common. This presentation describes U.S. Geological Survey (USGS) research to build KGs for integrating geospatial and non-spatial attribute semantics of topographic data. Those geographic information system databases are composed of various feature types and metadata attributes organized various themes and stored in different data formats, such as geodatabases, flat-file spreadsheets, and raster images. The system being created tests two research objectives: 1) the feasibility of semantic technology approaches for geospatial data within the context of national topographic data and 2) the contribution to building a body of knowledge about system architecture for geospatial ontologies and linked open data. This presentation discusses the context of topographic data semantics, the problem and aims of building the system, and the integrated KG framework. The basic workflow and operations of the system architecture consisting of open-source software are described. The architecture modifies existing software with unique solutions such as performing GeoSPAQL queries with Postgres, a relational table datastore, and a map interface with extensions to support linked data queries as browseable graphs. As public spatial data infrastructure, the system is made available as a Docker Container on GitHub.
\end{abstract}

